See Article page 1269.

\section{Commentary: The impact of hospital size on national trends and outcomes in isolated open proximal aortic surgery - does size matter?}

\author{
Marvin D. Atkins, MD, and Michael J. Reardon, MD
}

Proximal aortic disease, ranging from aneurysm to dissection, carries a high mortality without intervention. The surgical correction of proximal aortic pathology can present a technical challenge and, in many cases, requires advanced surgical techniques. How surgical experience plays into the outcomes in these cases is therefore of interest.

In this issue of the Journal, Hirji and colleagues tackle this question. ${ }^{1}$ The authors use the National Inpatient Sample (NIS) to identify patients older than 18 years of age who had an isolated proximal aortic procedure between 2002 and 2014. Proximal aortic surgery was defined as procedures involving the ascending aorta and arch. Patients with aortic valve replacement or repair, redo operations, endovascular procedures, descending thoracic aneurysm or thoracoabdominal aortic aneurysm procedures, or concomitant coronary artery bypass grafting were excluded. Over this 13-year period, 53,657 isolated open proximal aortic operations were identified. The authors' primary outcomes of interest were procedure volume and in-hospital mortality over this time period. Their secondary outcomes were complications such as myocardial infarction, stroke major bleeding, acute kidney injury, length of stay, costs, and disposition. They used hospital size based on number of beds as a surrogate for volume and hence experience. The NIS defines hospitals as small, medium, and large sizes based on the number of beds in a definition that varies slightly based

\footnotetext{
From the Department of Cardiovascular Surgery, Houston Methodist Hospital, Houston, Tex.

Disclosures: Dr Reardon serves as the national PI for the Arise EFS. Dr Atkins serves as the local PI on the Arise EFS.

Received for publication March 20, 2020; revisions received March 20, 2020; accepted for publication March 20, 2020; available ahead of print April 10, 2020.

Address for reprints: Michael J. Reardon, MD, Department of Cardiovascular Surgery, Houston Methodist Hospital, 6550 Fannin, Suite 1401, Houston, TX 77030 (E-mail: mreardon@ houstonmethodist.org).

J Thorac Cardiovasc Surg 2022;163:1279-80

$0022-5223 / \$ 36.00$

Copyright (c) 2020 by The American Association for Thoracic Surgery

https://doi.org/10.1016/j.jtcvs.2020.03.085
}

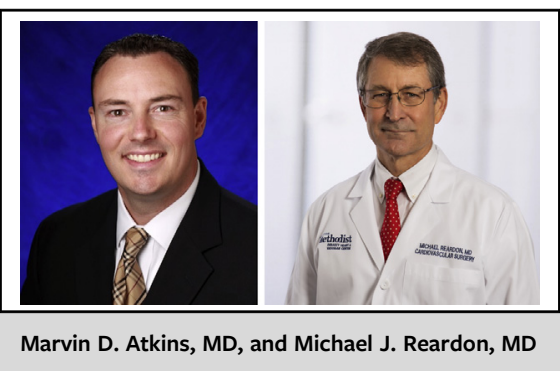

CENTRAL MESSAGE

Proximal aortic surgery volume is

increasing with a decrease in

mortality in nondissection cases

but no decrease for dissection.

Proximal aortic dissection re-

mains a morbid disease.

on region of the country (Northeast, Midwest, South, and West) and hospital type (rural, urban non-teaching, and urban teaching). Just more than $75 \%$ of cases were done in hospitals defined as large, which tended to have patients who were older, had more comorbidity, dissection, aneurysm, and patients with Marfan syndrome.

Over the period studied, the total number of cases increased from 3385 in 2002 to 4665 in $2014(P<.001)$ and mortality fell from $13.9 \%$ in 2002 to $6.7 \%$ in 2014 $(P<.001)$, based mainly on the mortality decrease in large hospital, but decreases in small/medium hospital did not vary over time. Mortality for dissection versus nondissection was $15.5 \%$ versus $6.6 \%$. One-third of the patients had aortic dissection, with mortality falling over time only in the nondissection group. Propensity score matching generated 9242 matched pairs, which showed no differences between large and non-large hospitals with respect to in-hospital mortality, myocardial infarction, stroke, major bleeding, and acute kidney injury. Their takeaway message was that there was an increase in procedure volume of $38 \%$ and decrease in mortality of $52 \%$ driven by nondissection cases. Larger hospitals had a shorter length of stay, lower cost, but high referral to rehabilitation centers.

We applaud the authors for this timely and comprehensive analysis. We would like to emphasize several points. The first is that aortic valve replacement and aortic valve repair were both excluded from the study, which would seem to eliminate all true aortic root surgery and leave us with only sinotubular junction-to-left subclavian procedures to consider. True aortic root surgery is likely 
to be concentrated in larger hospitals with aortic specialists. The NIS also does not allow one to distinguish between operations of the ascending aorta or aortic arch. One would presume that more complex arch operations requiring circulatory arrest are concentrated at large hospitals, further skewing the results. The second caveat of note is the greater discharge to rehabilitation units in the large hospital group. Since death outside of the hospital after 30 days would not count as mortality by the study definition, the greater referral to rehabilitation may have artificially lowered large hospital mortality. The high-risk surgical arm of the CoreValve high-risk randomized trial had an increase in the instantaneous hazard of death at 1 month that only decreased at month $4 .^{2}$ Analysis showed that most of these increased deaths were in rehabilitation centers, with failure to recover from the surgery.

Despite these issues, it is encouraging to see continued increase in proximal aortic cases with improving mortality in nondissection cases likely related to better surgical technique and care. Unfortunately, the mortality from ascending aortic dissection has not decreased. This matches what was seen in the recent review of Society of Thoracic Surgeons database cases, where the 30-day mortality for dissection repair of the ascending aortic alone versus with arch repair was $18.9 \%$ and $19 . \%{ }^{3}$ Ascending aortic dissection is a very morbid disease in which we strive to repair what is about to kill the patient without generally curing the dissection. We clearly have much work to do. Just as transcatheter aortic valve replacement has profoundly changed the options for treating severe symptomatic aortic stenosis in appropriate patient populations and thoracic endovascular aortic repair has caused a paradigm shift in the management of descending thoracic aortic disease, similar advances for ascending dissection may be around the corner.

The initial results from the early feasibility Arise Trial for stent graft treatment of ascending dissection were presented at the 2019 Society of Thoracic Surgeons meeting by Dr Roselli of the Cleveland Clinic. (Eric Roselli, MD, STS 2019, Late Breaking Abstract: Arise type A dissection early feasibility trial, Aortic session 1: dissection, January 27, 2019.) Although anatomic constraints will not allow all ascending dissection to be treated by stent graft, for appropriate patients this may represent a large step forward in an area in which we have not made significant advances in open surgical repair. The data generated in this study can serve as a baseline to compare future stent graft results. If stent graft technology can eventually be successfully applied to proximal aortic disease, bigger may well not be better. We look forward to future developments in this area.

\section{References}

1. Hirji S, Aranki S, McGurk S, Singh S, Pelletier M, Shekar P, et al. The impact of hospital size on national trends and outcomes in isolated open proximal aortic surgery. J Thorac Cardiovasc Surg. 2022;163:1269-78.e9.

2. Gaudiani V, Deeb GM, Popma JJ, Adams DH, Gleason TG, Conte JV, et al. Causes of death from the randomized CoreValve US pivotal high-risk trial. J Thorac Cardiovasc Surg. 2017;153:1293-301.e1.

3. Helder MRK, Schaff HV, Day CN, Pochettino A, Bagameri G, Greason KL, et al. Regional and temporal trends in the outcomes of repairs for acute type A aortic dissections. Ann Thorac Surg. 2020;109:26-33. 